\title{
Chemerin Promotes Mitogen-activated Protein Kinase / Extracellular Regulated Protein Kinases Activation to Induce Inflammatory Factor Production in Rat Synoviocytes
}

\section{chen wang}

Guangxi Medical University https://orcid.org/0000-0001-8923-8776

\section{Songrui Zhang}

Guangxi Medical University

\section{Longao Huang}

Guangxi Medical University

Jianyi Liu

Guangxi Medical University

\section{Gang Du}

Guangxi Medical University

Shan Lao ( $\nabla$ shanlaogx@163.com )

First Affiliated Hospital of Guangxi Medical University

\section{Research article}

Keywords: Chemerin, Osteoarthritis, Synoviocytes, Mitogen-activated protein kinase pathway

Posted Date: June 17th, 2021

DOl: https://doi.org/10.21203/rs.3.rs-546716/v1

License: (c) (i) This work is licensed under a Creative Commons Attribution 4.0 International License.

Read Full License 


\section{Abstract}

Aim

Chemerin is a chemokine from adipose tissue that specifically binds to the $\mathrm{G}$ protein-coupled receptor ChemR23 and has a chemotactic effect on macrophages and dendritic cells. A correlation between chemerin levels in synovial fluid and disease severity has been demonstrated in patients with osteoarthritis. However, the specific mechanism by which chemerin exerts its effects on osteoarthritis remains unclear. In this study, we investigated the mechanism of chemerin-associated synoviocyte inflammation.

Methods

Cell Counting Kit-8 (CCK-8) assays were used to identify concentrations of chemerin that had an effect on normal rat synoviocytes. The expression changes of mitogen-activated protein kinase kinase (MEK)/extracellular regulated protein kinases (ERK) pathway marker genes, including MEK, ERK, matrix metalloproteinase (MMP)-3 and MMP-13, were detected by fluorescence quantitative polymerase chain reaction (PCR). The phosphorylation of MEK, ERK1/2 and p38 mitogen-activated protein kinases (p38MAPK) by chemerin was analyzed by Western blotting, and the production of inflammatory factors after chemerin treatment was determined by enzyme-linked immunosorbent assay (ELISA). For in vivo assessment of chemerin function, rats were subjected to knee operation to provide a model for arthritis. The knees were then injected with normal saline or recombinant chemerin, and three weeks later, the synovium and knee joint tissue were harvested for HE staining observation and the synovial tissue was harvested for ELISA.

Results

Chemerin was demonstrated to enhance the proliferation of synoviocytes in a dose-dependent manner. The stimulatory effect of chemerin on synoviocytes was shown to involve the activation of MEK, ERK1/2 and p38, which was associated with the production of MMP-13, MMP-3, interleukin (IL)- 6 and IL-1 $\beta$ by synoviocytes. Inhibition of the ERK1/2 signaling pathway significantly inhibited chemerin-induced MMP13, MMP-3, IL- 6 and IL-1 $\beta$ production. HE staining showed that the degree of synovial hyperplasia and articular cartilage abrasion was more severe in chemerin-treated rats after knee operation. The articular cartilage surface was damaged, and the synovial tissue showed inflammatory cell infiltration. In rats that underwent operation without chemerin treatment, there was a slight inflammatory infiltration and higher levels of inflammatory factors as compared to unoperated rats; however, secretion of the downstream inflammatory factors IL-6, matrix metalloproteinases (MMP-3 and MMP-13) and IL-1 $\beta$ was significantly greater in the drug-treated group $(P<0.05)$.

Conclusion

Chemerin enhances the production of inflammatory factors in synoviocytes by activating the MEK/ERK signaling pathway. 


\section{Introduction}

Osteoarthritis (OA) is a chronic degenerative disease involving presentation of synovial lesions, which may be indicative of underlying inflammatory responses that precede or elude identification by X-ray imaging or Magnetic Resonance Imaging (MRI). ${ }^{1,2}$ The pathological process of OA involves inflammatory reaction of the synovium, which may manifest during the initial stages of synovitis as debilitating pain. Even if patients do not outwardly display clinical symptoms of synovitis, the diseased joints usually have local synovitis, and this inflammatory reaction is most significant in the adjacent articular cartilage injury area. ${ }^{3,4}$ The original theory was that obese patients are prone to $\mathrm{OA}$ because of cartilage destruction caused by biomechanical stress. ${ }^{5}$ However, more recent evidence suggests that a large amount of adipose tissue accumulates in obese patients, and that these tissues store energy and endocrine response factors, which can release a large number of cytokines and adipokines to participate in detrimental physiological and pathophysiological processes, such as immune and inflammatory responses, insulin resistance, and tumorigenesis. ${ }^{6}$ Consistently, caloric increase in animal models can lead to joint and systemic inflammatory response by promoting the release of adipokines, cytokines and chemokines. ${ }^{7}$ These findings underscore the importance of inflammatory responses in determining the occurrence and outcome of OA.

Chemerin is a recently identified chemotactic protein that guides macrophages and dendritic cells that express its receptor (chemokine like receptor 1 (CMKLR1); also known as ChemR23) ${ }^{8}$ to inflammatory sites and is involved in both adaptive and innate immunity. ${ }^{9}$ Chemerin is expressed in liver cells, white adipose tissue, monocyte-derived macrophages, and immature dendritic cells. ${ }^{10}$ In adipose tissues, chemerin is widely distributed and displays endocrine activity, with significant correlation in metabolic diseases such as obesity, type 2 diabetes, and cardiovascular disease. ${ }^{11-13}$ Chemerin levels in synovial fluid have been found to correlate with OA severity using the Kellgren-Lawrence grade as a criterion. ${ }^{14}$ Furthermore, ChemR23 is expressed in articular synoviocytes, ${ }^{15}$ and its expression is associated with the production of a variety of inflammatory mediators that are released in synovial lesions in OA and affect articular cartilage. ${ }^{3}$ Evidence suggests that the mitogen-activated protein kinase kinase (MEK)/extracellular regulated protein kinases (ERK) signaling pathway mediates inflammatory responses in $\mathrm{OA}$, and mitogen-activated protein kinases(MAPKs)have been evaluated as potential therapeutic targets. ${ }^{16,17}$ However, the mechanisms by which chemerin promotes inflammatory factor production in synoviocytes have not been evaluated. Therefore, in the present study, we investigated the activation of ERK/MEK signaling pathways by chemerin as well as the associated release of inflammatory mediators by joint synoviocytes. Our results provide a new approach for clinical treatment of OA through targeting of chemerin-associated Mitogen-activated protein kinase $\triangle M A P K \bigotimes$ signaling pathway.

\section{Material And Methods}

\section{1| Materials}


Rat synovial cells (RSC-364) were purchased from Shanghai Zeye Biotechnology Co., Ltd. Other experimental equipment and reagents included: Superclean cell bench (SW-CJ-2D model, Suzhou); ABI 7500 real-time PCR (RT-PCR) instrument (Applied Biosystems Inc, USA); constant temperature carbon dioxide incubator (Thermo Fisher Scientific, USA); Thermo microplate reader (Multiskan Fc); ultrasonic cell disruptor (Z-11D, Japan); Tanon 5200 Multi automatic chemiluminescence/fluorescence image analysis system (Tanon 5200 Multi); fluorescence inverted microscope (Olympus, Japan); 95\% Dulbecco's Modified Eagle's Medium (DMEM) high glucose medium (Solaibao, Beijing); cellular RNA reverse transcription kit (Thermo Fisher Scientific, USA); fluorescent quantitative Polymerase Chain Reaction(PCR) kit (Roche SYBR Green Master, Switzerland); recombinant murine chemerin (RampD, USA); trypsin-ethylene Diamine Tetraacetic Acid (EDTA) digestive solution (Solaibao, USA) Beijing); penicillin/streptomycin; Phosphate Buffered Saline (PBS) solution (Solaibao, Beijing); cell total RNA rapid extraction kit (Solaibao, Beijing); mycoplasma-free fetal bovine serum (Sijiqing, Fig. Zhejiang); High Performance Radio Immunoprecipitation Assay (RIPA) Lysis Solution (Biyuntian), Western and Immunoprecipitation IPQ Cell Lysis Solution (Biyuntian), Bicinchoninic acid (BCA) Protein Quantification Kit (Syndicate), Antibody electrochemiluminescence (ECL) kit (Syndicate), Immobilon-P $26.5 \times 3.75 \mathrm{~m}$ Roll polyvinylidene fluoride (PVDF) $0.45 \mu \mathrm{m}$ (Milipore), PageRuler Prejugated Protein Ladder, $10180 \mathrm{kDa}$ (Thermo), Spectra Multicolor Range High Activity Protein Ladder (Thermo), Horseradish Peroxidase (HRP)-Conjugated Antibody (Anti-rabbit glyceraldehyde 3-phosphate dehydrogenase (glyceraldehyde-3phosphate dehydrogenase $\mathrm{GGAPDH}$, Mouse IgG, HRP-linked antibody (Cell Signaling Technology (CST)), Anti-rabbit IgG, HRP-7074 (CST), Anti-ERK1 + ERK2 (abcam), Rabo-p44/42 MAPK (ERK1/2)T mAb (CST), p38 Rabbit mAb (CST), Phospho-p38 Rabbit mAb (CST); Cell Counting Kit-8 (CCK-8) Assay Kit (Dojindo); Rat Matrix Metalloproteinase (MMP)-3 Enzyme-Linked Immunosorbent Assay (ELISA) Kit, Rat MMP-13 ELISA Kit, Rat Tumor Necrosis Factor-a (TNF-a) ELISA Kit, Rat Interleukin (IL)-1 $\beta$ ELISA Kit, and Rat IL-6 ELISA Kit (enzyme-linked Biological, Shanghai).

\section{2 | Cell processing}

RSC-364 cells were cultured at $37^{\circ} \mathrm{C}$ in DMEM (10\% fetal bovine serum and $1 \%$ double antibody) in a $5 \%$ carbon dioxide incubator. The cells were subcultured by rinsing twice with sterile PBS followed by digestion in trypsin-EDTA (Solaibao, Beijing), which was evenly applied with observation under an inverted microscope. When $70-80 \%$ of the cells began to shrink and round or lift off the surface, $95 \%$ DMEM high-glucose culture medium was rapidly added, with gentle agitation using a sterile Papanicolaou dropper. The cells were subcultured at a ratio of 1:2 or 1:3. For cryopreservation, the cells were transferred into centrifuge tubes, the supernatants were removed after centrifugation, and the cell pellets were resuspended in an appropriate amount of cryopreservation solution (containing $20 \%$ fetal bovine serum, 10\% DMSO, and 70\% DMEM medium). The cells were dispensed into $1.5 \mathrm{~mL}$ cryopreservation tubes, placed in a programmed cryopreservation box in a $-80^{\circ} \mathrm{C}$ freezer, and then transferred to liquid nitrogen for long-term preservation. For recovering cryopreserved cells, cryotubes containing the cells were quickly placed in a $37^{\circ} \mathrm{C}$ water bath for re-warming. After thawing, the tubes were placed in a centrifuge $(1500 \mathrm{rpm}, 5 \mathrm{~min})$ to remove the supernatant. The cells were then re- 
suspended in DMEM culture medium and transferred to a culture flask. The rat synoviocytes were evaluated experimentally within two to five passages.

\section{3 | CCK-8 assay}

After RSC-364 synovial cells were passaged 3 times, the cells were seeded into two 96-well plates at a concentration of $5 \times 10^{3}$ cells/well. The plates were incubated in a carbon dioxide atmosphere at $37^{\circ} \mathrm{C}$ for $24 \mathrm{~h}$, then the plates were divided in half. Half of the plate was treated with MEK inhibitor $(10 \mu \mathrm{M}$ PD98059) for $30 \mathrm{~min}$; then, $100 \mu \mathrm{L}$ of $0,0.25,0.5$, or $1.0 \mu \mathrm{g} / \mathrm{mL}$ Reconstitute Chemerin's medium was added, and the plate was placed back in the incubator. After $24 \mathrm{~h}, 10 \mu \mathrm{L}$ of CCK-8 solution was added to each well according to the instructions of the CCK-8 kit (Dojindo); the plated was incubated in the cell culture chamber for another $4 \mathrm{~h}$. The absorbance was measured at $450 \mathrm{~nm}$ with a microplate reader. The experiment was repeated three times.

\section{4 | Quantitative RT-PCR}

The synoviocytes, passaged to the third generation, were seeded in a six-well plate at $4 \times 10^{4}$ cells/well and cultured for $24 \mathrm{~h}$. The cells were divided into two groups: the control group and the chemerin group $(0.5 \mu \mathrm{g} / \mathrm{mL}$ recombinant murine chemerin; RampD, USA). After continuous culture for $48 \mathrm{~h}$, total RNA was extracted according to the instructions of the RNA rapid extraction kit (Solaibao, Beijing), and then cDNA was synthesized by reverse transcription (Thermo Fisher Scientific, USA) and quantified using a fluorescence quantitative PCR kit (Roche SYBR Green Master, Switzerland). Quantitative PCR was performed for GAPDH, MMP-3, MMP-13, MEK, and ERK using the primers in Table 1. GAPDH was set as the internal reference. All experimental assays were repeated three times.

Table 1

Primer sequences for PCR.

\begin{tabular}{|lll|}
\hline Gene & Forward primer $\left(\mathbf{5}^{\prime} \sim \mathbf{3}^{\prime}\right)$ & Reverse primer $\left(\mathbf{3}^{\prime} \sim \mathbf{5}^{\prime}\right)$ \\
\hline MEK & AACGGTCAGAAAGTGGCGAT & ACGTTCTTTCGGCAGGTCAT \\
\hline Mrk & TCTGCAGTTAACGGGACCAG & AGCTCTAGCTCCTCCAGCTT \\
\hline MMP-13 & TGCAGAACTGTGGGAAGCCA & ACCGTACCTCCAAGACGTCT \\
\hline GADPH & TCCACCACCC TGTTGCTGTA & CCCTTGTTCCTCACCCACAC \\
\hline
\end{tabular}

\section{5 | Western blot analysis}

The cells, which were passaged for three generations, were divided into the control and chemerin groups and were seeded in six-well plates at $4 \times 10^{4} /$ well. After $24 \mathrm{~h}$ of attachment, the cells were stimulated for 10 min and then RIPA lysis buffer (containing protease inhibitors) was added. After incubation on ice, the cells were lysed by sonication and centrifuged, and the supernatant was recovered. The protein concentrations were determined by BCA assay, and equal amounts of protein were mixed with loading 
buffer in a boiling water bath for $5 \mathrm{~min}$. Electrophoresis was carried out at $80 \mathrm{~V}$ initially, and then $120 \mathrm{~V}$ until the bromophenol blue had migrated to about $1 \mathrm{~cm}$ away from the lower edge of the gel. The membranes were blocked with rocking at room temperature for $1 \mathrm{~h}$ and then washed three times with Tris-buffered saline Tween (TBST) for 5 min each. The corresponding antibodies were diluted according to the manufacturer recommendations (HRP-Conjugated GAPDH Antibody, HRP-60004, Fig. Proteintech; Anti-Mouse IgG, HRP-linked Antibody, 7076P2, CST; Anti-rabbit IgG, HRP-linked Antibody, 7074S, CST; Anti-ERK1 + ERK2, ab184699, Abcam; Phospho-p44/42 MAPK (ERK1/2) Rabbit mAb, 4377T, CST; Rabbit mAb, 8690T, CST; Phospho-p38 Rabbit mAb, 4511T, CST). The membranes were incubated with primary antibody at room temperature for $2 \mathrm{~h}$ or overnight at $4^{\circ} \mathrm{C}$ and then washed 3 times with TBST for 5 min each time and incubated with HRP-labeled secondary antibody for $1 \mathrm{~h}$ at room temperature. Chromogenic droplets were added to the membranes, and the gray values of the target bands were calculated with Image $\mathrm{J}$ software.

\subsection{Enzyme linked immunosorbent assay}

After RSC-364 synoviocytes were cultured for 3 passages, the cells were seeded in a six-well plate at $4 \times$ $10^{4} /$ well and cultured in a $37^{\circ} \mathrm{C}$ constant temperature incubator for $24 \mathrm{~h}$. They were divided into three groups: the control group, the chemerin group $(0.5 \mu \mathrm{g} / \mathrm{mL}$ chemerin) and the inhibitor group. The inhibitor group was pre-treated with MEK inhibitor (10 $\mu$ M PD98059, CST \# 9900S) for $1 \mathrm{~h}$ and then recombinant chemerin was added at the same concentration as in the chemerin group. After $48 \mathrm{~h}$, the supernatant was collected to determine the levels of MMP-3, MMP-13, TNF-a, IL-1 $\beta$ and IL- 6 secreted by synoviocytes of rats in each group according to ELISA kit (Shanghai) instructions.

\section{7 | Animal handling:}

Thirty sterile SD rats were divided into three groups. Groups B and C were first modeled by the modified Huths anterior cruciate ligament cutting method ${ }^{23}$. After the operation, the rats were intraperitoneally injected with 200,000 units of penicillin for 3 consecutive days and were permitted to roam freely in the cage. One week after modeling, 20 rats were randomly divided into two groups. In group C, each knee joint was injected with about $0.1 \mathrm{ml}$ solution containing recombinant Chemerin, once every three days for three weeks; group B was injected with the same amount of normal saline. In group A, no surgical modeling was performed, and each knee joint was injected with the same amount of normal saline at the same times as the above two groups. The growth environment of the three groups of rats was the same.

\section{8 | HE staining and ELISA}

Three weeks after the final chemerin or saline injection, the rats were injected with an overdose of $1 \%$ sodium pentobarbital and sacrificed. The skin was incised along the middle of the knee joint until the entire knee joint was exposed. An incision was made from the upper edge of the patella to the femur, and then the soft tissue was separated from the tibia with ophthalmic scissors along both sides of the patella. The knee joint cavity was opened, and forceps were used to excise the patella and surrounding tissues. The synovial tissue of the patella, which appeared as a layer of smooth light and pale yellow that continued downwards under the patella, was removed and carefully cut with ophthalmic scissors. HE 
staining was performed as follows: First, paraffin sections were deparaffinized with xylene and washed

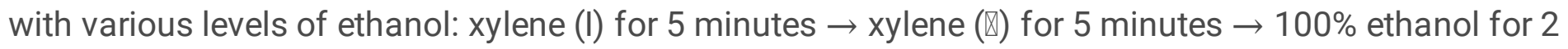
minutes $\rightarrow 95 \%$ ethanol for 1 minute $\rightarrow 80 \%$ ethanol for 1 minute $\rightarrow 75 \%$ Ethanol for 1 minute $\rightarrow$ distilled water for 2 minutes. Then, the sections were stained with hematoxylin dye solution for 10 minutes and rinsed with autoclaved water. Differentiating solution was applied for 30 seconds, followed by warm water (about $50^{\circ} \mathrm{C}$ ) for 5 minutes, eosin dye solution for 2 minutes, and a final rinse with autoclaved water. Conventional ethanol dehydration was performed step by step ( $95 \%$ ethanol (I) for 1 minute $\rightarrow 95 \%$ ethanol (II) for 1 minute $\rightarrow 100 \%$ ethanol (I) for 1 minute $\rightarrow 100 \%$ ethanol (II) for 1 minute $\rightarrow$ two Toluene carbolic acid (3:1) for 1 minute $\rightarrow$ xylene (I) for 1 minute $\rightarrow$ xylene (II) for 1 minute), and then the sections were sealed with neutral resin, covered with a cover slip, and observed under a microscope.

For ELISA, synovial tissue was excised according to the above method and was added to sterile PBS (1 $\mathrm{g}: 10 \mathrm{ml}$ ) and mashed. The suspension was centrifuged at $1000 \times \mathrm{g}$ for 10 minutes, and the supernatant was recovered. The levels of MMP-3, MMP-13, IL-1 $\beta$ and IL- 6 in the synovial tissues were determined according to the instructions of the ELISA kit (enzyme-linked immunosorbent assay, Shanghai).

\section{9 | Statistical analysis}

SPSS 25.0 software was used for statistical processing, expressed as $x \pm s$. One-way Anova analysis of variance was used for multiple group comparisons. T tests were used for comparisons. The difference was considered statistically significant when $P<0.05$.

\section{Results}

\section{1 | Chemerin regulates the proliferation of synovial cells in a dose-dependent manner}

To verify the effect of chemerin on synovial cells, we established a RSC-364 cell culture. As expected, the cells exhibited elongated polygonal shapes (Fig. 1). We performed CCK-8 analysis after $24 \mathrm{~h}$ of treatment with different concentrations of recombinant mouse chemerin. The results showed that after $24 \mathrm{~h}$, chemerin promoted proliferation, and the proliferation of chemerin could be prevented by pretreating the cells with the MEK/ERK inhibitor PD98059 (Fig. 2). The difference in the effects of chemerin at $24 \mathrm{~h}$ suggests that the effects of chemerin may be time-and dose-dependent. In addition, the results support the use of $0.5 \mu \mathrm{g} / \mathrm{mL}$ chemerin as a dose that affects synovial cells in $24 \mathrm{~h}$ in subsequent experiments, and further indicates that the effect of chemerin may be related to the MEK/ERK pathway.

\section{2 | Chemerin promotes the activation of MAPK pathways in synoviocytes}


Based on the above findings and given the important role of MAPK pathways in the pathogenesis of $\mathrm{OA},{ }^{16,17}$ we sought to determine whether chemerin may regulate the expression of MAPKs. After $48 \mathrm{~h}$, the gene expression of MEK and ERK in synoviocytes that were cultured in the presence of $0.5 \mu \mathrm{g} / \mathrm{mL}$ recombinant mouse chemerin were significantly increased compared to the levels in the control group. Further, the expression of MMP-3 and MMP-13 genes also increased (Fig. 3).

MAPK activity is often also regulated by phosphorylation. Therefore, we also evaluated the total and phosphorylated levels of MAPK proteins after 10 min of exposure of the synoviocytes to chemerin. Western blot analysis demonstrated a significant increase in expression of total ERK1/2, pERK1/2, MEK, pMEK and p-p38 after chemerin treatment, though levels of total p38 were unaltered (Fig. 4). These findings are consistent with our mRNA results and indicate that chemerin may regulate cell growth by activating ERK1/2 expression and p38 phosphorylation.

\section{3 | Chemerin promotes the expression of inflammatory cytokines in a MAPK-dependent manner}

Given the role of synovial tissue inflammation in OA progression, ${ }^{3,4}$ we evaluated whether chemerin may modulate the production of inflammatory mediators in synoviocytes, and whether MAPKs may be involved in this process. Cells were stimulated with chemerin for $48 \mathrm{~h}$ in the presence of absence of the MEK/ERK pathway inhibitor PD98059, and ELISA assays were performed to evaluate the concentrations of MMP-3, MMP-13, TNF- $a$, IL-1 $\beta$, and IL- 6 in culture supernatants. The results demonstrate that chemerin induced a significant increase in each of these inflammatory modulators, and that the increase in MMP-3 and MMP-13 was abolished in synoviocytes pretreated with PD98059 (Fig. 5). Levels of TNF-a, IL-1 $\beta$, and IL-6 also appeared to be reduced by PD98059, and the decrease did reach statistical significance. These results are consistent with a role for the MEK/ERK pathway in mediating the activation of MMPs and potentially other inflammatory mediators in synoviocytes.

\subsection{IChemerin promotes synovial inflammatory hyperplasia and the release of inflammatory factors in rats}

To determine whether the inflammatory effects of chemerin are recapitulated in an in vivo model, we divided rats into three groups. One group (the "blank group") did not receive surgery and received injections of saline. The second group (the "control" group) received knee surgery to induce arthritis followed by saline injections, and the third group (the "chemerin" group) received knee surgery followed by chemerin injections. Observation of HE-stained synovial tissue under a microscope showed that the tissue from the blank group contained 1-2 layers of synovial cells arranged regularly and with a mostly flat shape (Fig. 6). The tissue was relatively loose, with a small amount of capillaries and no obvious inflammatory cell infiltration; the cartilage surface was smooth and flat, without damage or bone destruction, and no inflammatory cell infiltration could be seen under the microscope. For the control group (Fig. 7), the synovial tissue was more hyperplasic than that of the blank group. A small amount of 
synovial fibroblasts proliferation was observed, and inflammatory cell infiltration could be seen in the intercellular space. There was slight cartilage destruction, but no obvious bone destruction. Finally, in the chemerin group (Fig. 8), the synovial tissue showed obvious signs of proliferation, and the infiltration of inflammatory cells (mostly lymphocytes) was more than that of the control group. There was also obvious vascular proliferation, and the continuity of the articular cartilage surface was interrupted, with obvious cartilage defects.

ELISA results verified that recombinant rat chemerin can also promote synovial tissue to produce corresponding inflammatory mediators in animal experiments. Compared with the control group, each of the assayed inflammatory factors in the chemerin group showed a significant increase. In addition, when the control group was compared with the blank group, there was corresponding synovial inflammation, but the degree of lesions and the degree of inflammatory factors were lower than for the drug group (see Fig. 9). These differences are statistically significant $(P<0.05)$, and the results are consistent with the role of synovial cell MMPs and potential other inflammatory mediators in the activation of synovial cells under the action of chemerin.

\section{Discussion}

Recent studies have demonstrated that chemerin is an adipokine with chemotactic activity, and that its levels, as well as the levels of other inflammatory factors, are higher in obese individuals. ${ }^{18}$ Chemerin promotes the secretion of TNF, IL-1 $\beta, I L-6, M M P-1$, and MMP-8 by chondrocytes, and these factors play an important role in the development of OA. ${ }^{19}$ Furthermore, chemerin has been detected in the synovial fluid of $\mathrm{OA}$ patients, and its expression is associated with the levels of inflammatory factors. ${ }^{20}$ Therefore, we hypothesized that chemerin may regulate inflammatory function in synoviocytes.

Our results provide direct evidence that chemerin promotes the production of inflammatory mediators in synoviocytes. This was demonstrated by an ELISA of a variety of inflammatory cytokines and chemokines, including MMP-3, MMP-13, TNF- $a, \mathrm{IL}-1 \beta$, and IL-6. A previous study suggests that systemic MMP-13 may play a role in knee $O A$ and may be regulated by inflammatory signaling, ${ }^{21}$ thus supporting the relevance of our findings. Furthermore, TNF- $\alpha$, IL-1 $\beta$ and IL- 6 have each been shown to mediate an increase in the expression level of the receptor for chemerin (Chem23), ${ }^{8}$ which suggests the possibility of a positive feedback loop that may exacerbate inflammation in $\mathrm{OA}$ and other inflammatory conditions. In addition, our results demonstrated that chemerin promotes synovial inflammatory hyperplasia, cartilage damage, and increases in corresponding inflammatory cells and inflammatory factors during in vivo experiments. While the pathogenesis of OA ultimately involves the degeneration of fragments and particles of cartilage, inflammatory responses in the synovium, including inflammatory mediators released by synovial lesions, are known to aggravate articular cartilage destruction, contributing to a vicious cycle that leads to disease progression. ${ }^{3}$ Therefore, our results support a model in which chemerin functionally interacts with inflammatory factors and amplifies their effect in mediating cartilage degeneration. 
MAPK signaling also is known to play an important role in the pathogenesis of $O A .{ }^{16,17,22}$ In this study, we found that chemerin activates MAPK expression in synoviocytes at both the transcriptional and posttranscriptional level. In addition, pretreatment with MAPK pathway-specific inhibitors attenuated the induction of MMP-3, MMP-13, TNF- $a$, IL-1 3 , and IL- 6 by chemerin. These results indicate that chemerin may induce or exacerbate the development of OA by regulating the MEK/ERK signaling pathway, thereby affecting the secretion of inflammatory factors by synoviocytes.

In summary, chemerin plays an important role in obesity-induced $O A$, and our evidence suggests that its function may involve the activation of MAPK signaling in synoviocytes, which leads to the production of inflammatory mediators that ultimately cause cartilage degeneration. Our findings support the potential of chemerin to serve as a biomarker of disease severity, though additional research will be needed to assess its value in the clinical monitoring and intervention of early $\mathrm{OA}$ in obese patients.

\section{Declarations}

\section{Author contributions}

Conceptualization:Shan Lao. Experimental design: Chen Wang®Songrui Zhang. Date analysis: Chen

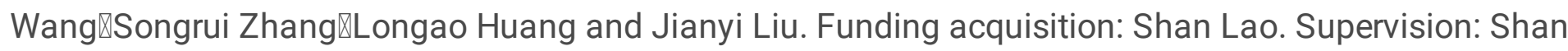
Lao $₫$ Gang Du. All authors participated in interpreting the data, in writing and critically reviewing the manuscript, and approved the final version.

\section{Acknowledgments}

This work was supported by the National Natural Science Foundation of China [grant number 8156090038]; the Natural Science Foundation of Guangxi Zhuang Autonomous Region [grant number 2017GXNSFAA198159]; and the Scientific Research Project of Guangxi Zhuang Autonomous Region [grant number AB19110030]. We thank LetPub (www.letpub.com) for its linguistic assistance and scientific consultation during the preparation of this manuscript.

\section{Conflicts of Interest}

The authors declare that there is no conflict of interest regarding the publication of this paper.

\section{Animal experiment ethics statement:}

All animal experiments in this experiment have complied with the ARRIVE guidelines, and comply with the "British Animal (Scientific Procedure) Act of 1986" and the relevant EU guidelines on animal experiments Directive 2010/63/EU, or the National Institutes of Health on nursing Guidelines and Use of Laboratory Animals (NIH Publication No. 8023, revised in 1978)

\section{Data Availability Statements $\square$}


The author believes that all data is already in the supplementary material.

\section{References}

1. Crema MD, Roemer FW, Marra MD, Guermazi A. MR imaging of intra- and periarticular soft tissues and subchondral bone in knee osteoarthritis. Radiol Clin N Am. 2009;47(4):687-701.

2. Loeuille D, Rat AC, Goebel JC, et al. Magnetic resonance imaging in osteoarthritis: which method best reflects synovial membrane inflammation? Osteoarthr Cartil. 2009;17(9):1186-92.

3. Ayral X, Pickering EH, Woodworth TG, Mackillop N, Dougados M. Synovitis: a potential predictive factor of structural progression of medial tibiofemoral knee osteoarthritis-results of a 1 year longitudinal arthroscopic study in 422 patients. Osteoarthr Cartil. 2005;13(5):361-7.

4. Benito MJ. Synovial tissue inflammation in early and late osteoarthritis. Ann Rheum Dis. 2005;64(9):1263-7.

5. Dahik VD, et al. "Rewiring of Lipid Metabolism in Adipose Tissue Macrophages in Obesity: Impact on Insulin Resistance and Type 2 Diabetes." International journal of molecular sciences vol. 21,15 5505. 31 Jul. 2020.

6. Deng Y, Scherer PE. Adipokines as novel biomarkers and regulators of the metabolic syndrome. Ann N Y Acad Sci. 2010;1212(1):E1-19.

7. Pita J, Panadero A, Soriano-Guillén L, Rodríguez E, Rovira A. The insulin sensitizing effects of PPAR- $Y$ agonist are associated to changes in adiponectin index and adiponectin receptors in Zucker fatty rats. Regul Pept. 2012;174(1-3):18-25.

8. Mariani F, Roncucci L. Chemerin/chemR23 axis in inflammation onset and resolution. Inflammation Res. 2015;64(2):85-95.

9. Wittamer Vr, Franssen JD, Vulcano M, et al. Specific recruitment of antigen-presenting cells by chemerin, a novel processed ligand from human inflammatory fluids. J Exp Med. 2003;198(7):97785 .

10. Bluher M, Rudich A, Kloting N, et al. Two patterns of adipokine and other biomarker dynamics in a long-term weight loss intervention. Diabetes Care. 2012;35(2):342-9.

11. Stojek M. The role of chemerin in human disease. Postepy Hig Med Dosw. 2017;71(1):110-7.

12. Sitar-Taut AV, Coste SC, Tarmure S, et al. Diabetes and obesity-cumulative or complementary effects on adipokines, inflammation, and insulin resistance. J Clin Med. 2020;9(9):2767.

13. Kaur J, Mattu HS, Chatha K, Randeva HS. Chemerin in human cardiovascular disease. Vasc Pharmacol. 2018;110:1-6.

14. Huang K, Du G, Li L, Liang H, Zhang B. Association of chemerin levels in synovial fluid with the severity of knee osteoarthritis. Biomarkers. 2012;17(1):16-20.

15. Kaneko K, Miyabe Y, Takayasu A, et al. Chemerin activates fibroblast-like synoviocytes in patients with rheumatoid arthritis. Arthritis Res Ther. 2011;13(5):,R158. 
16. Saklatvala J. Inflammatory signaling in cartilage: MAPK and NF-kappaB pathways in chondrocytes and the use of inhibitors for research into pathogenesis and therapy of osteoarthritis. Curr Drug Targets. 2007;8(2):305-13.

17. Loeser RF, Erickson EA, Long DL. Mitogen-activated protein kinases as therapeutic targets in osteoarthritis. Curr Opin Rheumatol. 2008;20(5):581-6.

18. Buechler C, Feder S, Haberl E, Aslanidis C. Chemerin isoforms and activity in obesity. Int J Mol Sci. 2019;20(5):1128.

19. Huss RS, Huddleston JI, Goodman SB, Butcher EC, Zabel BA. Synovial tissue-infiltrating natural killer cells in osteoarthritis and periprosthetic inflammation. Arthritis Rheum. 2010;62(12):3799-805.

20. Zhao L, Yamaguchi Y, Ge XM, et al. Chemerin 156F, generated by chymase cleavage of prochemerin, is elevated in joint fluids of arthritis patients.[. J]Arthritis Res Ther. 2018;20:132.

21. Ruan G, Xu J, Wang $K$, et al. Associations between knee structural measures, circulating inflammatory factors and MMP13 in patients with knee osteoarthritis. Osteoarthr Cartil. 2018;26(8):1063-9.

22. Mengshol JA, Vincenti MP, Coon $\mathrm{Cl}$, Barchowsky A, Brinckerhoff CE. Interleukin-1 induction of collagenase 3 (matrix metalloproteinase 13) gene expression in chondrocytes requires p38, c-jun $\mathrm{N}$ terminal kinase, and nuclear factor kB: Differential regulation of collagenase 1 and collagenase 3 . Arthritis Rheum. 2000;43(4):801-11.

23. Rogart JN, Barrach HJ, Chichester CO.. Articular collagen degradation in the Hulth-Telhag model of osteoarthritis[J]. Osteoarthritis \& Cartilage, 1999, 7(6):p. 539.

\section{Figures}
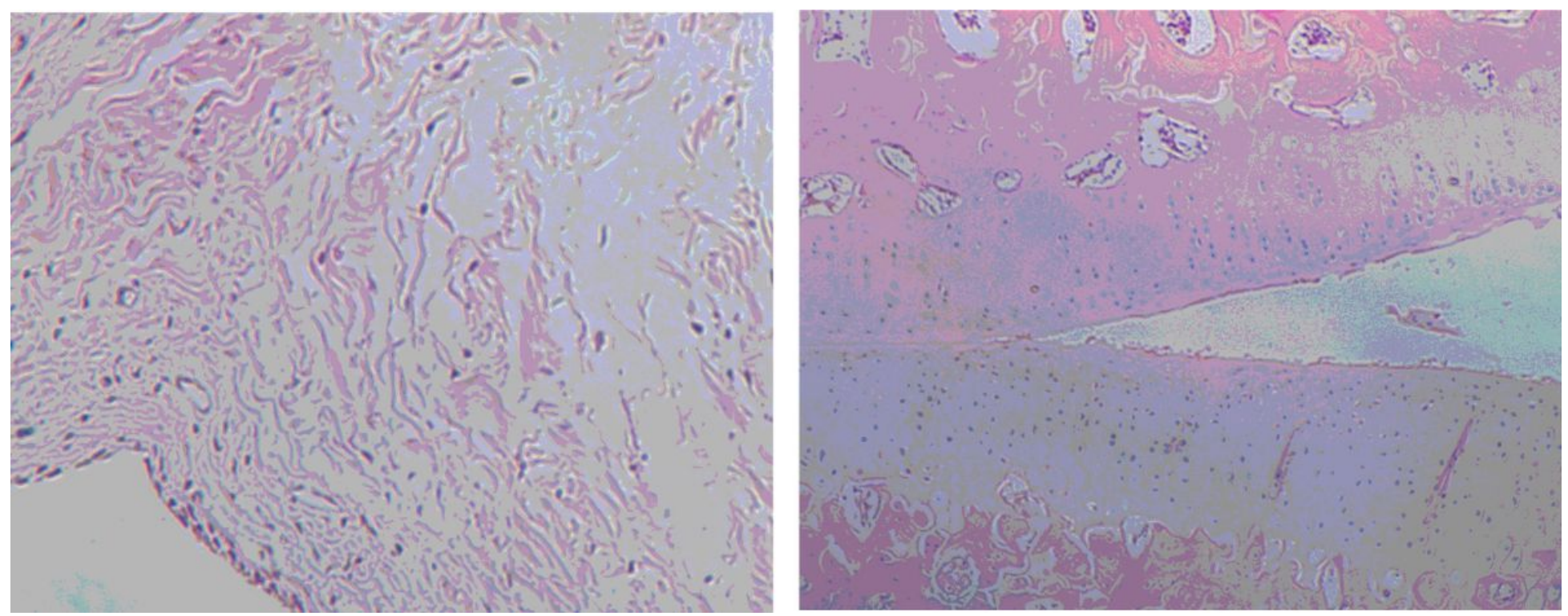

\section{Figure 1}

The morphology of RSC-364 synoviocytes. (a) 40X. (b) 100X. Abbreviations: RSC-364, rat synovial cells 

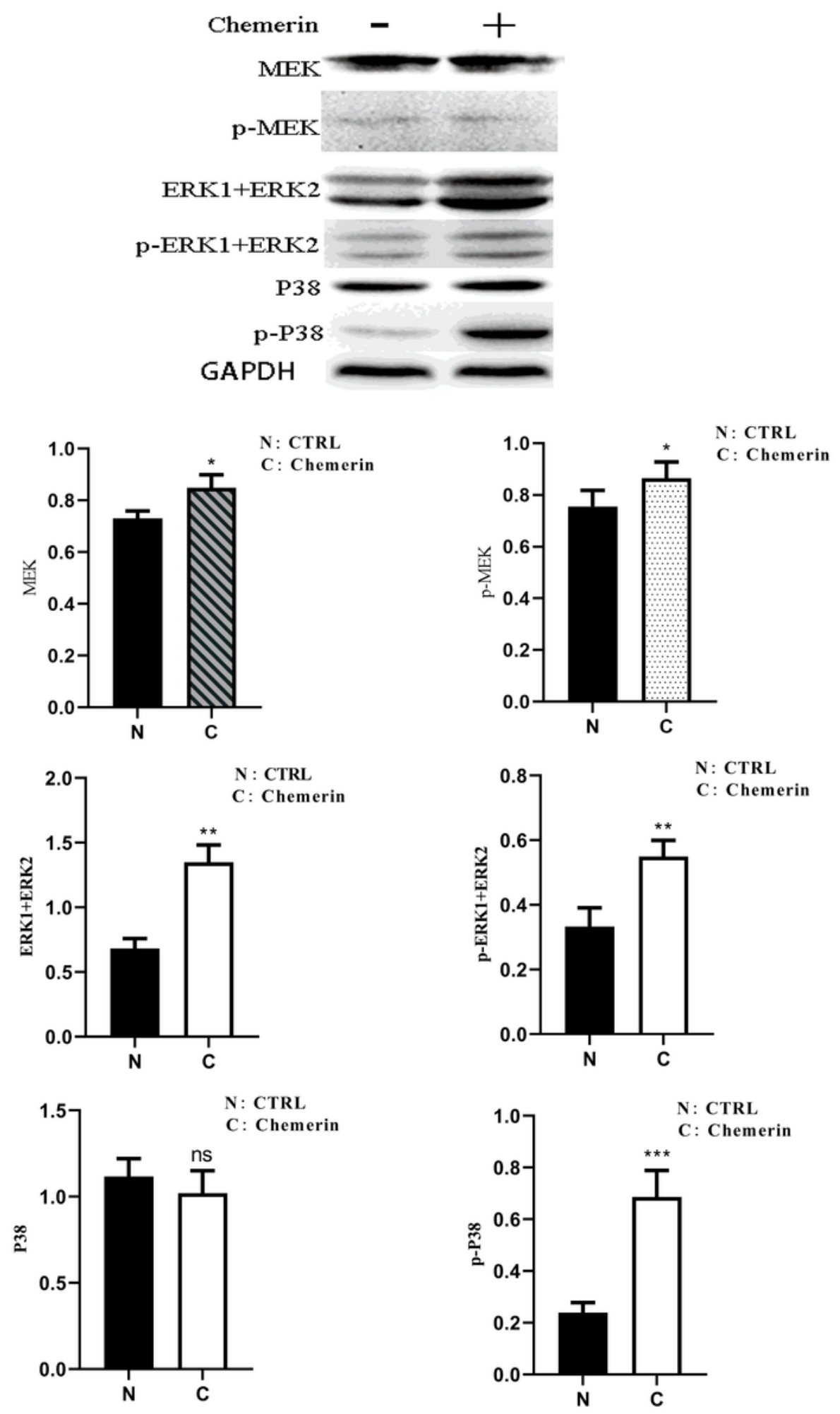

Figure 2

Chemerin affects the proliferation of RSC-364 Proliferation was measured by the CCK-8 assay. Cells were pre-treated with the MEK/ERK pathway inhibitor PD98059 where indicated prior to chemerin treatment for $24 \mathrm{~h}$. ${ }^{\star \star \star \star}$ indicates $\mathrm{P}<0.01$ for the chemerin group compared to the control group; ${ }^{\star *}$, ${ }^{\star \star \star}$ indicates a statistical difference between the inhibitor group and the chemerin group at $\mathrm{P}<0.05$ or $\mathrm{P}<0.01$; $* *$ indicates a statistical difference between the treatment groups at $P<0.05$. Results are mean \pm SD of 3 
independent experiments. Abbreviations: RSC-364, rat synovial cells; CCK-8, cell counting kit-8; MEK, mitogen-activated protein kinase kinase; ERK, extracellular regulated protein kinases; SD, standard deviation

A

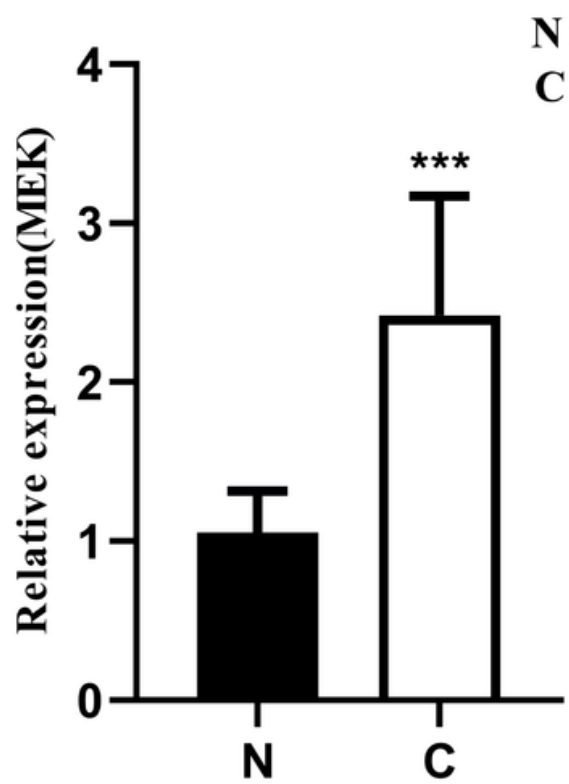

C

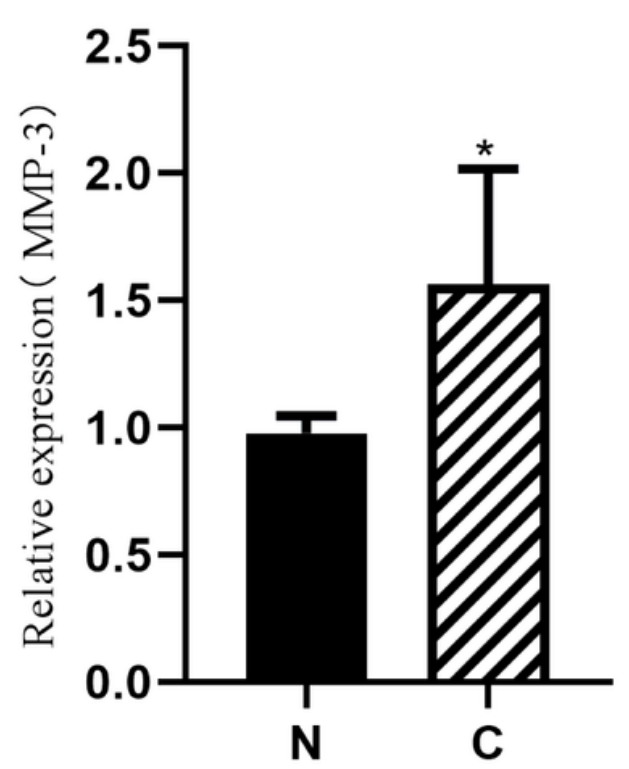

B

N: CTRL

C: Chemerin

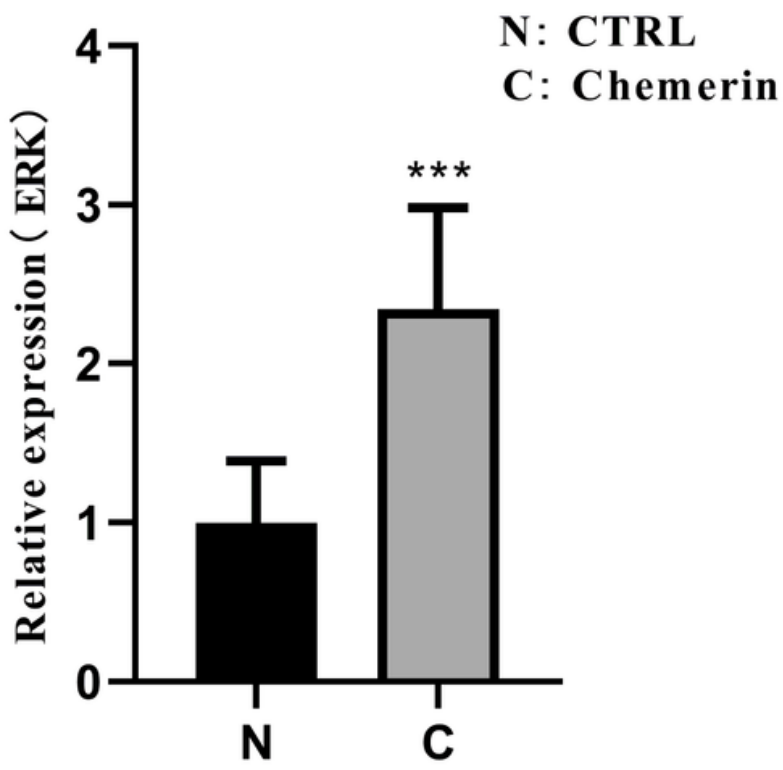

D

N: CTRL

$C$ : Chemerin

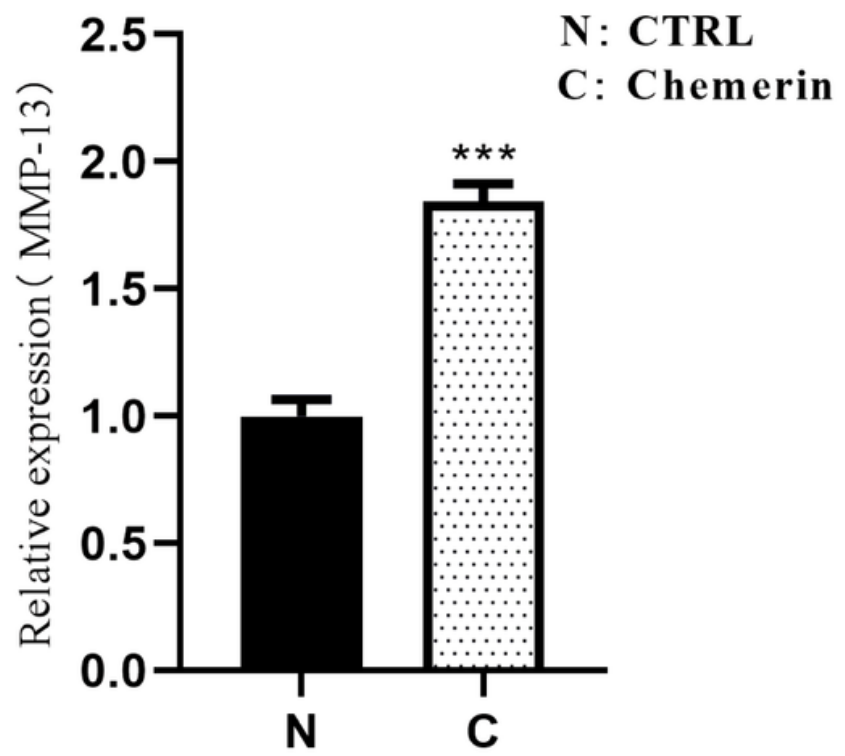

Figure 3

Chemerin activates MAPK pathway gene expression in RSC-364 synoviocytes. The expression of MAPK pathway genes was measured by RT-PCR and normalized to the expression of GAPDH in control synovial cells $(\mathrm{N})$ and synovial cells exposed to $0.5 \mu \mathrm{g} / \mathrm{mL}$ chemerin for $48 \mathrm{~h}$ (C). (a) Relative expression of MEK mRNA. (b) Relative expression of ERK mRNA. (c) Relative expression of MMP-3 mRNA. (d) Relative 
expression of MMP-13 mRNA. *** compared with the CTRL (N) group, $\mathrm{P}<0.05$ is statistically different. The result is the mean \pm SD of 3 independent experiments. Abbreviations: MAPK, mitogen-activated protein kinases; RSC-364, rat synovial cells; RT-PCR, real-time PCR; GAPDH, glyceraldehyde 3-phosphate dehydrogenase; ERK, extracellular regulated protein kinases; MMP, Matrix Metalloproteinase; CTRL, control; SD, standard deviation
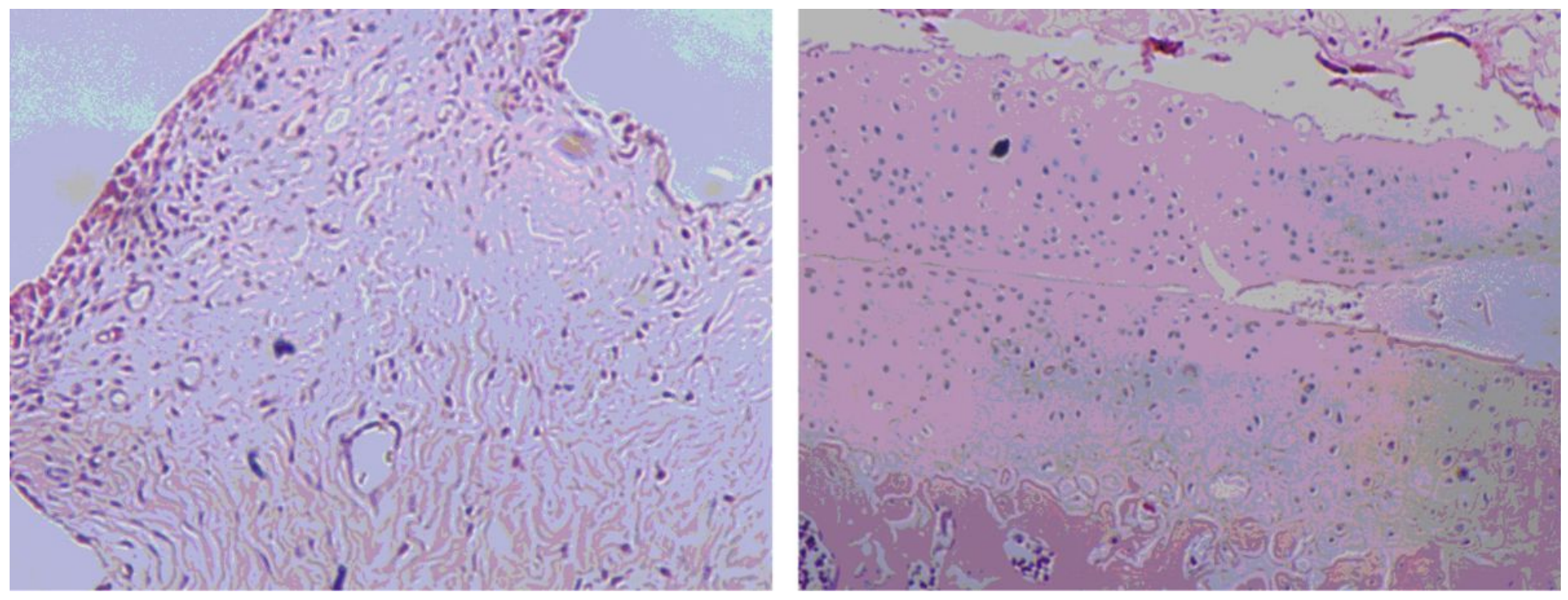

\section{Figure 4}

Chemerin activates the expression and phosphorylation of MAPK pathway proteins in RSC-364 synoviocytes. Western blot analysis was performed for synoviocytes that were either unstimulated or

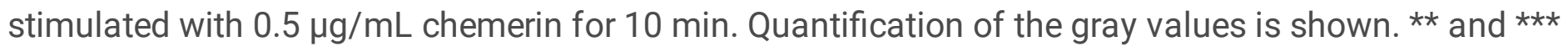
indicate $\mathrm{P}<0.05 ; \mathrm{ns}=$ not significant. Results are representative of 3 independent experiments. Abbreviations: MAPK, mitogen-activated protein kinases; RSC-364, rat synovial cells 
A

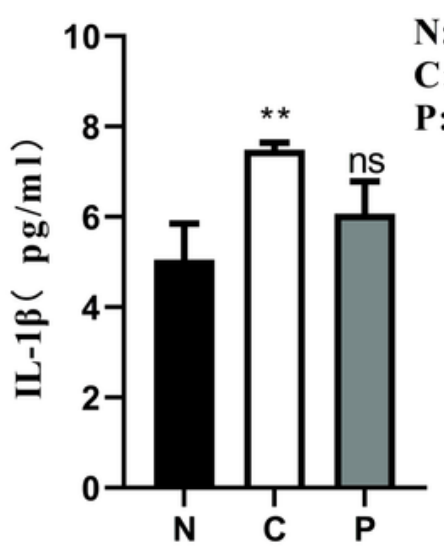

C

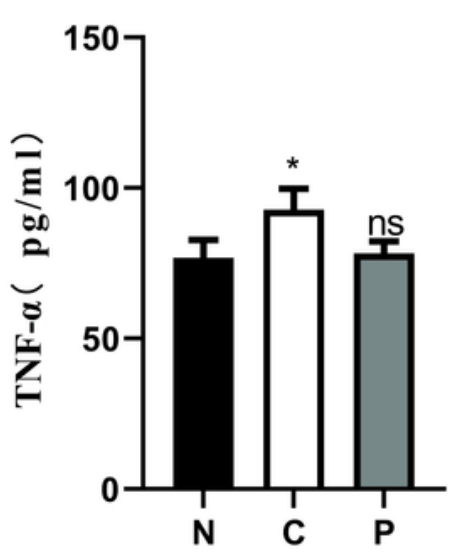

B

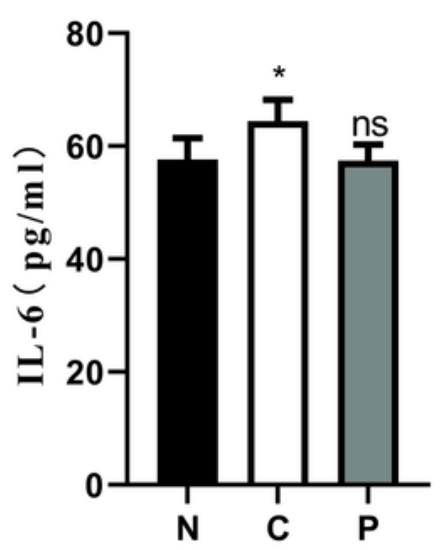

$\mathrm{N}:$ CTRL

C:Chemerin

P:Chemerin+PD98059
N:CTRL

$C:$ Chemerin

P:Chemerin+PD98059

D

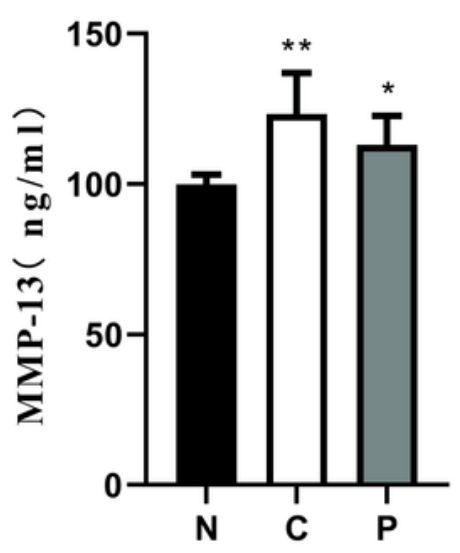

N:CTRL

C:Chemerin

P:Chemerin+PD98059
E

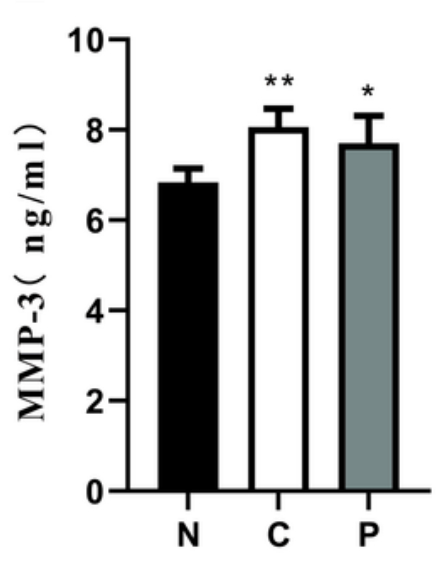

N:CTRL

C:Chemerin

P:Chemerin+PD98059

Figure 5

Chemerin enhances the secretion of inflammatory factors in RSC-364 synoviocytes in a MAPK-dependent manner. The levels of MMP-3, MMP-13, TNF- $\alpha$, IL-1 $\beta$ and IL- 6 in synovial cell supernatants was determined by ELISA after $48 \mathrm{~h}$ culture in the presence or absence of chemerin and the MEK inhibitor PD98059. * $* \star$ indicate comparison with $\mathrm{N}$ group: $\mathrm{P}<0.05$, ns indicates that it is not obvious compared with the $\mathrm{N}$ group. ${ }^{*},{ }^{* *}$ Indicates the addition of drug group and blocker group: $\mathrm{P}<0.05$. Results are mean 
+ SD of 3 independent experiments. Abbreviations: RSC-364, rat synovial cells; MAPK, mitogen-activated protein kinases; MMP, Matrix Metalloproteinase; TNF-a, Tumor Necrosis Factor-a; IL, Interleukin; ELISA, enzyme-linked immunosorbent assay; SD, standard deviation

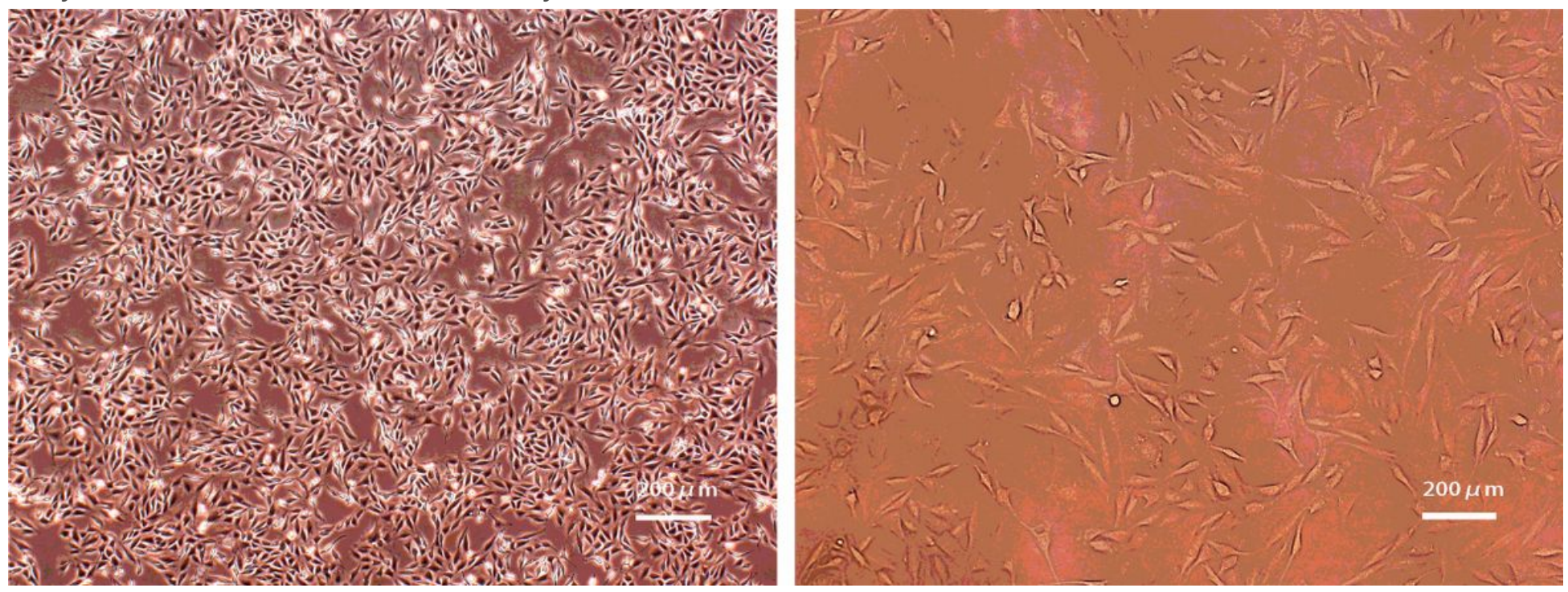

\section{Figure 6}

Microscopic observation of synovial tissue and cartilage from the blank group of rats. The blank group of rats were not subjected to surgery and were injected with saline. A) synovial tissue microscopic observation; the tissue was relatively loose; B) cartilage microscopic observation; the cartilage surface was smooth.
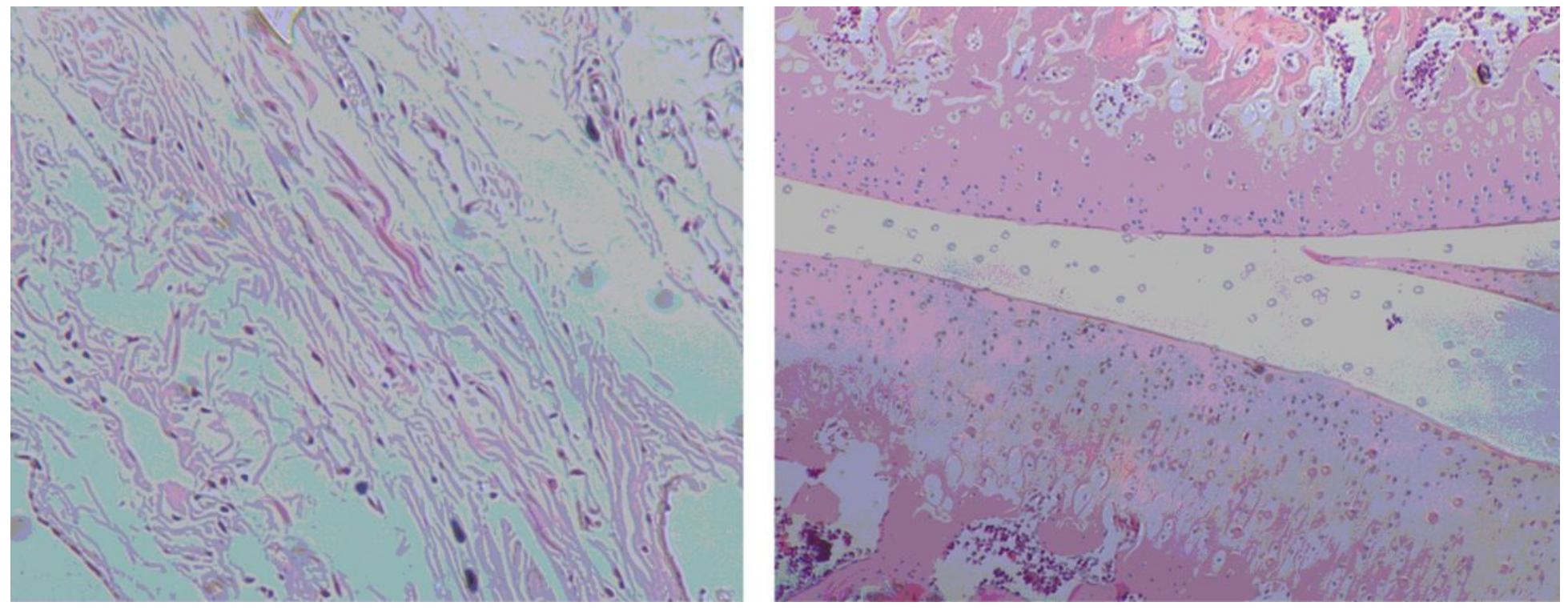

\section{Figure 7}

Microscopic observation of synovial tissue and cartilage from the control group of rats. The control group of rats were subjected to knee surgery and then injected with saline. A) synovial tissue microscopic observation; there were more fibroblast synovial cells than for the blank group; B) cartilage microscopic observation; the cartilage surface was slightly damaged. 


\section{$* * * *$}

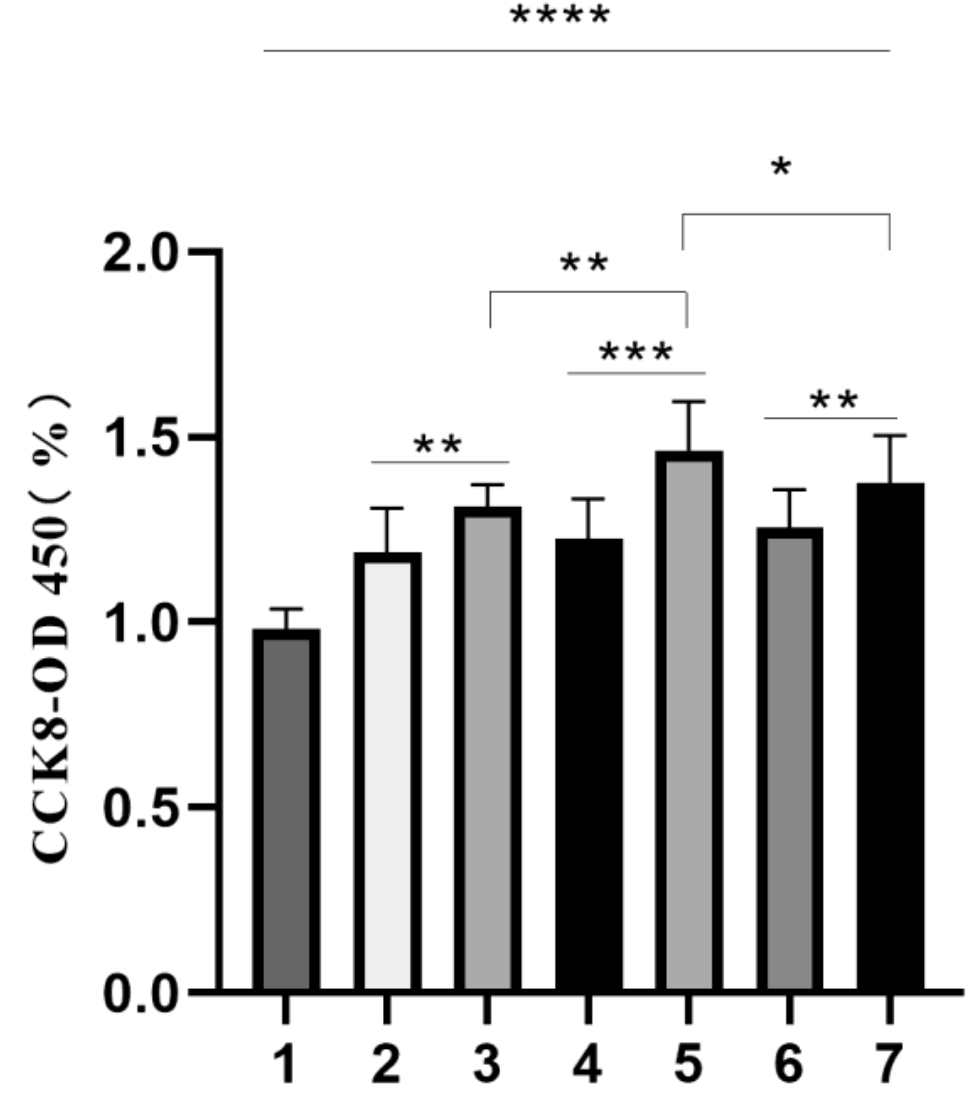

1:CTRL

2:0.25 $\mu \mathrm{g} / \mathrm{mlChemerin}+\mathrm{PD}$

3:0.25 $\mu \mathrm{g} / \mathrm{mlChemerin}$

$4: 0.5 \mu \mathrm{g} / \mathrm{mlChemerin}+\mathrm{PD}$

$5: 0.5 \mu \mathrm{g} / \mathrm{mlChemerin}$

6: $1 \mu \mathrm{g} / \mathrm{mlChemerin}+\mathrm{PD}$

$7: 1 \mu \mathrm{g} / \mathrm{mlChemerin}$

Figure 8

Microscopic observation of synovial tissue and cartilage from the chemerin group of rats. The chemerin group of rats were subjected to knee surgery and then injected with chemerin. A) synovial tissue microscopic observation; the proliferation of fibroblasts was obvious, and there was lymphocyte infiltration; B) cartilage microscopic observation; the cartilage surface defect could be seen. 
A

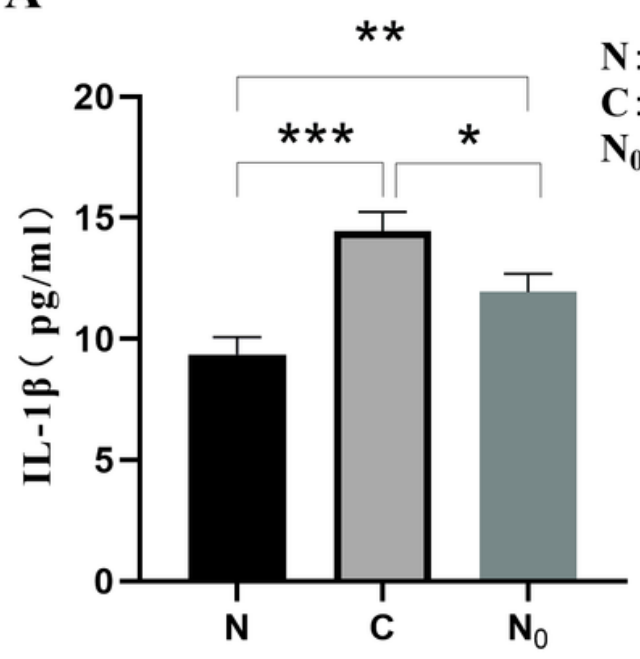

C

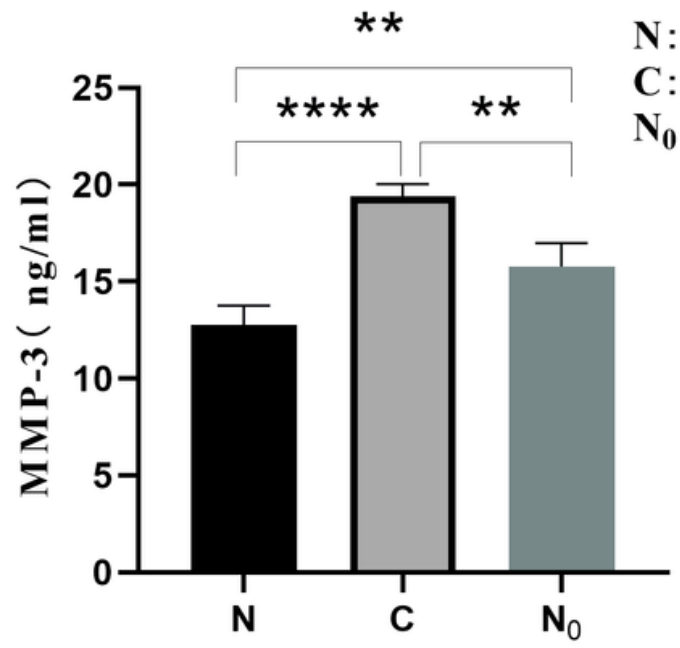

B

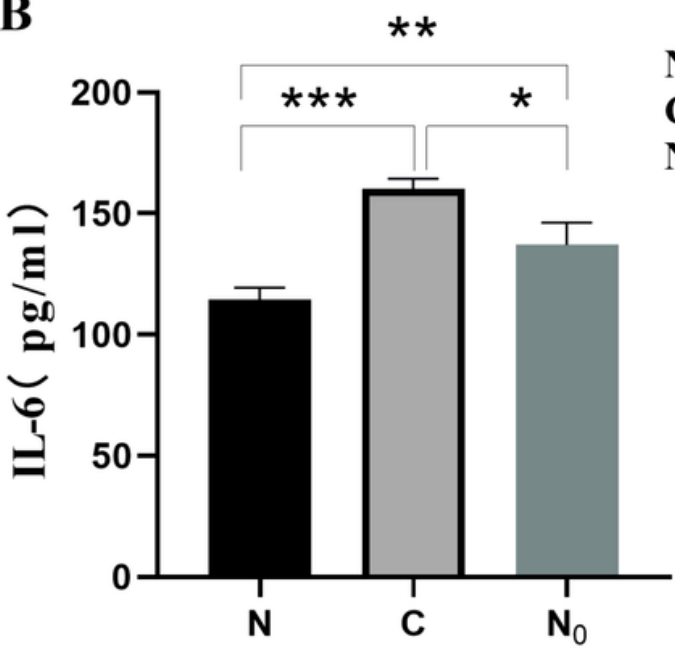

D

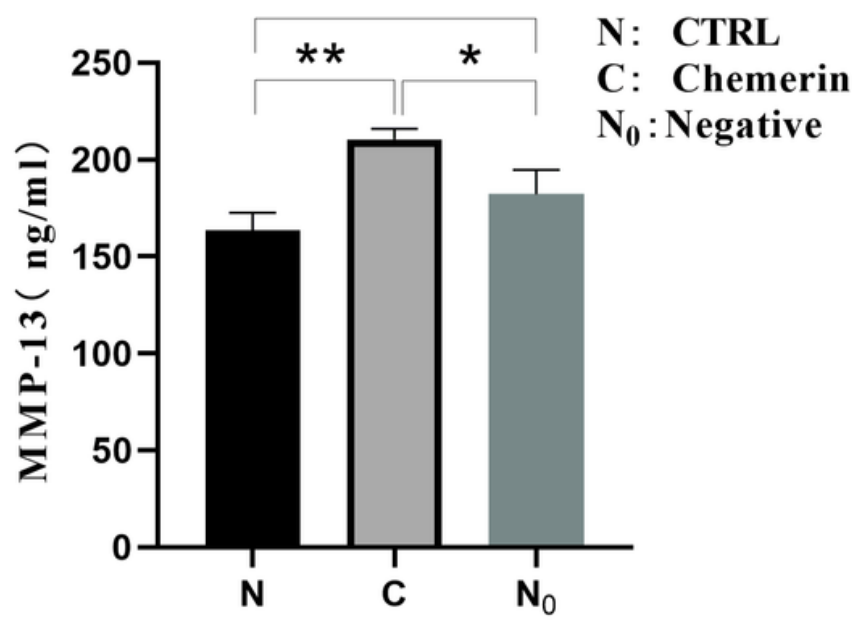

Figure 9

Inflammatory factor levels in rats after knee surgery and/or chemerin injection. $\mathrm{N}$ represents the blank group (injection of saline only), C represents the medication group (surgery + drugs), and NO represents the control group (surgery + saline). Levels of inflammatory factors were determined by ELISA. * ** indicates comparison with group $\mathrm{N}: \mathrm{P}<0.05$; $\star \star \star$ indicates comparison with group $\mathrm{N}$ : $\mathrm{P}<0.01$. The result is the mean \pm+ standard deviation of 3 independent experiments. 